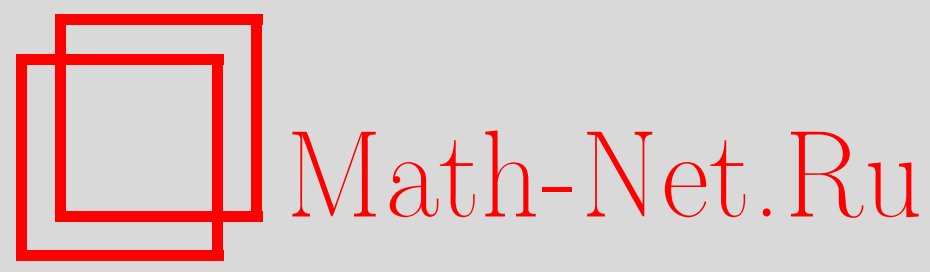

Л. А. Бассалыго, В. А. Зиновьев, О многочленах специального вида над конечным полем нечетной характеристики, достигающих границы Вейля, Матем. заметки, 2005, том 78, выпуск 1, 16-25

DOI: https://doi.org/10.4213/mzm2556

Использование Общероссийского математического портала Math-Net.Ru подразумевает, что вы прочитали и согласны с пользовательским соглашением http://www . mathnet.ru/rus/agreement

Параметры загрузки:

IP: 3.85 .7 .115

26 апреля 2023 г., 16:03:58

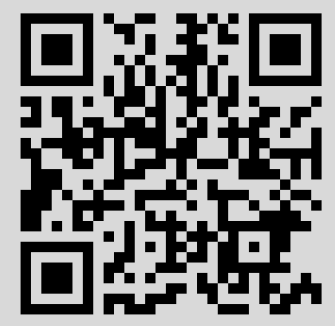




\title{
О МНОГОЧЛЕНАХ СПЕЦИАЛЬНОГО ВИДА НАД КОНЕЧНЫМ ПОЛЕМ НЕЧЕТНОЙ ХАРАКТЕРИСТИКИ, ДОСТИГАЮЩИХ ГРАНИЦЫ ВЕЙЛЯ
}

\author{
Л. А. Бассалыго, В. А. Зиновьев
}

Явная конструкция многочленов над конечньм полем нечетной характеристики, модуль тригонометрических сумм которых достигает границы Вейля, основана на построении циклических матриц заданного ранга. При изучении таких матриц существенную роль играют многочлены Диксона второго рода.

Библиограффия: 5 названий.

1. Введение. Пусть $q=p^{\ell}, Q=q^{m}=p^{\ell m}$, где $p$ - простое число, $\ell, m$ - целые положительные числа, $m \geqslant 2$, и $F_{p} \subseteq F_{q} \subset F_{Q}$ - соответствующие конечные поля. Пусть далее

$$
\begin{gathered}
f(x)=a_{1} x+\cdots+a_{n} x^{n}, \quad a_{i} \in F_{Q}, \\
S(f)=\sum_{x \in F_{Q}} \exp \left\{\frac{2 \pi i \operatorname{Tr}_{Q}(f(x))}{p}\right\},
\end{gathered}
$$

где

$$
\operatorname{Tr}_{Q}(x)=x+x^{p}+x^{p^{2}}+\cdots+x^{p^{l m-1}}, \quad x \in F_{Q} .
$$

Известная оценка Вейля [1] утверждает, что модуль тригонометрической суммы ограничен сверху:

$$
|S(f)| \leqslant(\operatorname{deg} f-1) \sqrt{Q}
$$

Мы продолжаем начатое в [2] изучение $|S(f)|$ для многочленов $f(x)$ специального вида:

$$
f(x)=\sum_{s=0}^{\lfloor m / 2\rfloor} a_{s} x^{1+q^{s}}, \quad a_{s} \in F_{Q}, \quad \operatorname{Tr}_{Q}(f(x)) \neq \text { const } .
$$

В [2] был предложен общий подход к этой проблеме, заключающийся в сведении тригонометрической суммы многочлена $f(x)$ вида (3) от одной переменной над полем $F_{q^{m}}$ к кратной тригонометрической сумме квадратичной формы от $m$ переменных над полем $F_{q}$ (точнее, для любого многочлена вида (3) строилась квадратичная форма с тем

Работа выполнена при частичной финансовой поддержке Российского фонда фундаментальных исследований, грант № 03-01-00098, и фонда INTAS, грант № 00-738. 
же значением тригонометрической суммы). Все нетривиальные $\left(\neq 0, q^{m}\right)$ значения, которые может принимать модуль кратной тригонометрической суммы квадратичной формы от $m$ переменных, известны (см. [1], [3]), и в [2] полностью или частично описаны многочлены вида (3) с максимальным и минимальным модулями тригонометрической суммы. И описание, и полученные результаты сушественно разнятся для полей четной и нечетной характеристики. В [4] мы описали подкласс многочленов вида (3) с максимальньм значением модуля тригонометрической суммы для полей четной характеристики. В настоящей работе мы проделываем аналогичный путь для полей нечетной характеристики, так что всюду далее $p$ - нечетное простое число.

Итак, известно [1], [3], что модуль тригонометрической суммы квадратичной формы от $m$ переменных над полем нечетной характеристики $F_{q}$ может принимать одно из следующих значений:

$$
q^{m-r / 2}, \quad r=1,2, \ldots, m
$$

и никаких других нетривиальных значений принимать не может. Следовательно, то же самое относится и к модулю тригонометрической суммы многочленов вида (3), которые в дальнейшем, воспользовавшись нечетностью характеристики поля, всегда будем представлять в следующем виде:

$$
f(x)=a_{0} x^{2}+2 \sum_{s=1}^{\lfloor m / 2\rfloor} a_{s} x^{1+q^{s}} .
$$

По представлению (5) построим квадратную матрищу $B=\left[b_{i j}\right]$ размера $m \times m$ :

$$
\begin{array}{ll}
b_{i i}=a_{0}^{q^{i}}, & i=0,1, \ldots, m-1, \\
b_{i j}=b_{j i}=a_{j-i}^{q^{i}}+a_{i-j}^{q^{j}}, & i \neq j,
\end{array}
$$

где индексы у коэффициентов $a_{s}$ рассматриваются по модулю $m\left(a_{s}=0\right.$, если $s>$ $\lfloor m / 2\rfloor)$.

Утверждение 1 из [2] позволяет вычислить $|S(f)|$ по рангу матрицы $B$.

УТВЕРЖДЕНИЕ 1. Пусть $f(x)$ - произвольный многочлен вида (5) и матрица В определяется соотношениями (6). Тогда

$$
|S(f)|=q^{m-(1 / 2) \operatorname{rank}(B)} .
$$

Сравнение этого утверждения и (4) показывает, что модуль тригонометрической суммы многочлена вида (5) принимает значение $q^{m-r / 2}, r=1, \ldots, m$, тогда и только тогда, когда $\operatorname{rank}(B)=r$. Интересньй вопрос заключается в том, как для любого $r$, $r=1, \ldots, m$, явно описать многочлены вида (5) минимальной степени, модуль тригонометрической суммы которых равен $q^{m-r / 2}$. Действительно, если степень такого многочлена равна $q^{t}+1$ (т.е. $a_{t} \neq 0, a_{t+1}=\cdots=a_{\lfloor m / 2\rfloor}=0$ ), то оценка Вейля $(2)$ для него равна

$$
q^{t+m / 2}=q^{m-(m-2 t) / 2},
$$

и она достигается тогда и только тогда, когда ранг соответствующей матрицы $B$ равен $m-2 t$. Итак, задача явного описания многочленов вида (5) степени $q^{t}+1(t=1,2$, 
$\ldots,\lfloor m / 2\rfloor)$, достигающих оценки Вейля, сводится к явному описанию матрищ вида (6) с $a_{t} \neq 0, a_{t+1}=\cdots=a_{\lfloor m / 2\rfloor}=0$, ранг которых равен $m-2 t$. Для произвольного $t$ эта задача, видимо, достаточно сложна, но в некоторых частных случаях при дополнительных ограничениях на коэффициенты многочленов ответ может быть найден. Так как этот ответ зависит от четности числа $m$, далее случаи нечетного и четного $m$ рассматриваются отдельно.

2. Случай $m=2 k+1, k \geqslant 2$. В работе [2] мы изучили два крайних случая: $t=k$ (т.е. ранг матрицы $B$ равен 1 ) и $t=1$ (т.е. ранг матрицы $B$ равен $2 k-1$ ). В первом случае мы описали все такие матрищы, т.е. все многочлены вида $(5)$ степени $q^{k}+1$, достигаюшие границы Вейля [2, теорема 1]. Во втором мы описали все такие матрищы, т.е. все многочлены вида (5) степени $q+1$, достигающие границы Вейля, при условии, что все коэффищиенты многочлена принадлежат полю $F_{q}$, а не полю $F_{q^{m}}$ [2, теорема 2]. Теперь мы рассмотрим случай, когда $t=k-1$ (т.е. матрица $B$ имеет ранг 3 ), при том же условии на коэффициенты: $a_{0}, a_{1}, \ldots, a_{k-1}$ принадлежат полю $F_{q}$. Тогда первые четыре строки матрицы $B$ имеют вид $\left(a_{k-1} \neq 0\right)$

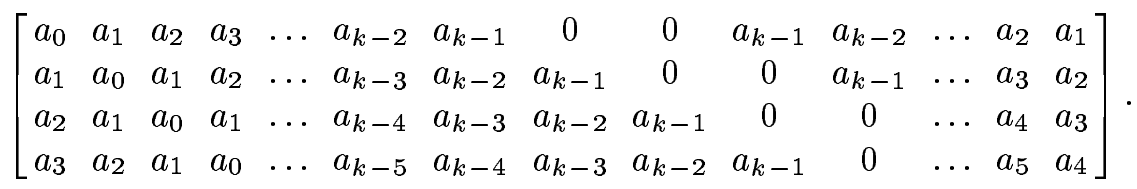

Если четвертая строка матрицы $B$ является линейной комбинацией первых трех строк, то ее ранг равен 3 , так как матрица $B$ циклическая, а ее первые три строки линейно независимы. Обозначим через $b_{i}$ строку $B$ с номером $i$. Четвертая строка $b_{4}$ матрицы $B$ является линейной комбинацией первых трех тогда и только тогда, когда найдутся элементы $\lambda_{1}, \lambda_{2}, \lambda_{3} \in F_{q},\left(\lambda_{1}, \lambda_{2}, \lambda_{3}\right) \neq(0,0,0)$, такие, что

$$
b_{4}=\lambda_{1} b_{1}+\lambda_{2} b_{2}+\lambda_{3} b_{3}
$$

Обозначим через $B_{4}$ матрицу размера $4 \times(2 k+1)$, образованную первыми четырьмя строками матрищы $B$. Рассматривая столбцы матрицы $B_{4}$ с двумя нулями (т.е. столбцы с номерами $k+2, k+3$ и $k+4)$, из соотношения (7) находим, что

$$
\begin{aligned}
a_{k-2} & =\lambda_{3} a_{k-1}, \\
a_{k-1} & =\lambda_{1} a_{k-1}, \\
0 & =\lambda_{1} a_{k-2}+\lambda_{2} a_{k-1} .
\end{aligned}
$$

Отсюда получаем (так как $a_{k-1} \neq 0$ )

$$
\lambda_{1}=1, \quad \lambda_{2}=-\lambda_{3} .
$$

Полагая $\lambda_{2}=\lambda$, перепишем соотношение (7) в следующем виде:

$$
b_{1}=-\lambda b_{2}+\lambda b_{3}+b_{4} .
$$


С учетом (9) последние $k-2$ столбцов матрицы $B_{4}$ позволяют выразить все коэффициенты $a_{i}, i=1, \ldots, k-2$, через $a_{k-1}$ и $\lambda$ с помощью следующих рекуррентных соотношений:

$$
\begin{aligned}
a_{k-2} & =-\lambda a_{k-1} \\
a_{k-3} & =-\lambda a_{k-2}+\lambda a_{k-1} \\
a_{k-4} & =-\lambda a_{k-3}+\lambda a_{k-2}+a_{k-1} \\
a_{k-5} & =-\lambda a_{k-4}+\lambda a_{k-3}+a_{k-2} \\
\ldots \ldots & \ldots \ldots \ldots \ldots \ldots \ldots \ldots \ldots \ldots \ldots \ldots \ldots \ldots \ldots \ldots \ldots \\
a_{i} & =-\lambda a_{i+1}+\lambda a_{i+2}+a_{i+3}, \quad i=1, \ldots, k-4 .
\end{aligned}
$$

Рассмотрим теперь первые четыре столбца матрицы $B_{4}$ (остальные столбцы дают те же соотношения, что и (10)). Нетрудно видеть, что первьй столбец порождает то же соотношение, что и четвертьй, а второй - то же, что и третий. Из первого столбца $B_{4}$ вытекает, что рекуррентное соотношение (10) имеет место и при $i=0$.

Итак, все коэффициенты $a_{i}, i=0,1, \ldots, k-2$, выражаются через $a_{k-1}$ и $\lambda$, и осталось еще одно соотношение, порождаемое вторым столбцом матрицы $B_{4}$ :

$$
a_{2}=(1-\lambda) a_{1}+\lambda a_{0}
$$

Это соотношение легко переписывается в простом эквивалентном виде.

УТВЕРЖДЕНИЕ 2. Пусть $G_{k}(x)$ задается следующим рекуррентным соотношением:

$$
G_{k}(x)=x G_{k-1}(x)-G_{k-2}(x), \quad k \geqslant 2,
$$

$2 \partial e$

$$
G_{0}(x)=1, \quad G_{1}(x)=x-1 .
$$

Тогда соотношение (11) әквивалентно соотношению

$$
G_{k}(1+\lambda)=0
$$

ДокАЗАТЕЛЬСТво. Используя (13), запишем соотношение (11) таким образом:

$$
G_{0}(1+\lambda) \cdot a_{2}+\left(G_{1}(1+\lambda)-G_{0}(1+\lambda)\right) \cdot a_{1}-G_{1}(1+\lambda) \cdot a_{0}=0 .
$$

Далее, воспользовавшись соотношением для $a_{0}$ из (10) и рекурсией $(12)$, перепишем соотношение (15) в следующем виде:

$$
G_{1}(1+\lambda) \cdot a_{3}+\left(G_{2}(1+\lambda)-G_{1}(1+\lambda)\right) \cdot a_{2}-G_{2}(1+\lambda) \cdot a_{1}=0 .
$$

Продолжая подобным образом, получаем, что при любом $i, i=0,1, \ldots, k-1$, соотношение (11) эквивалентно следующему соотношению:

$$
G_{i}(1+\lambda) \cdot a_{i+2}+\left(G_{i+1}(1+\lambda)+G_{i}(1+\lambda)\right) \cdot a_{i+1}-G_{i+1}(1+\lambda) \cdot a_{i}=0
$$

(здесь полагаем $a_{k+1}=a_{k}=0$ ).

Отсюда при $i=k-1$ следует утверждение, так как $a_{k-1} \neq 0$. 
Обозначение $G_{k}(x)$ в рекурсии (12) с начальными условиями (13) выбрано не случайно. Оно заимствовано из книги [5, гл. 2, с. 32], где так обозначается разность двух соседних многочленов Диксона второго рода с параметром, равным 1 (так как вообще все приводимые нами сведения о многочленах Диксона взяты из упомянутой книги [5, гл. 2], далее мы это не оговариваем особо; впрочем, все эти сведения могут быть легко проверены непосредственно). Вообще говоря, многочлены Диксона зависят от переменной $x$ и параметра $a$, но нам понадобятся лишь многочлены с параметром $a$, равным 1 , и чтобы не загромождать формулы, мы этот параметр опускаем. Многочлен Диксона второго рода $E_{k}(x)$ степени $k$ с параметром 1 определяется следующим образом:

$$
E_{k}(x)=\sum_{i=0}^{\lfloor k / 2\rfloor}(-1)^{i}\left(\begin{array}{c}
k-i \\
i
\end{array}\right) x^{k-2 i}
$$

и он удовлетворяет рекуррентному соотношению (12) с начальными условиями

$$
E_{0}(x)=1, \quad E_{1}(x)=x
$$

По определению

$$
G_{k}(x)=E_{k}(x)-E_{k-1}(x)
$$

и поэтому

$$
G_{k}(1+\lambda)=\sum_{i=0}^{\lfloor k / 2\rfloor}(-1)^{i}\left(\begin{array}{c}
k-i \\
i
\end{array}\right)(1+\lambda)^{k-2 i}-\sum_{i=0}^{\lfloor(k-1) / 2\rfloor}(-1)^{i}\left(\begin{array}{c}
k-1-i \\
i
\end{array}\right)(1+\lambda)^{k-1-2 i} .
$$

ЗАмЕчАниЕ 1 . Формула (20) имеет любопытную графовую интерпретацию (возможно, эта интерпретация известна). Рассматривается плоский графф-решетка, ребра которого по одной оси имеют длину 1 , а по другой - длину 2 , и на каждом ребре длины 2 посредине добавляется новая вершина (так что степень каждой новой вершины, как и корневой, равна 2, степень вершин, расположенных на осях, равна 3 и остальных вершин равна 4). Этот граф изображен ниже на рис. 1.

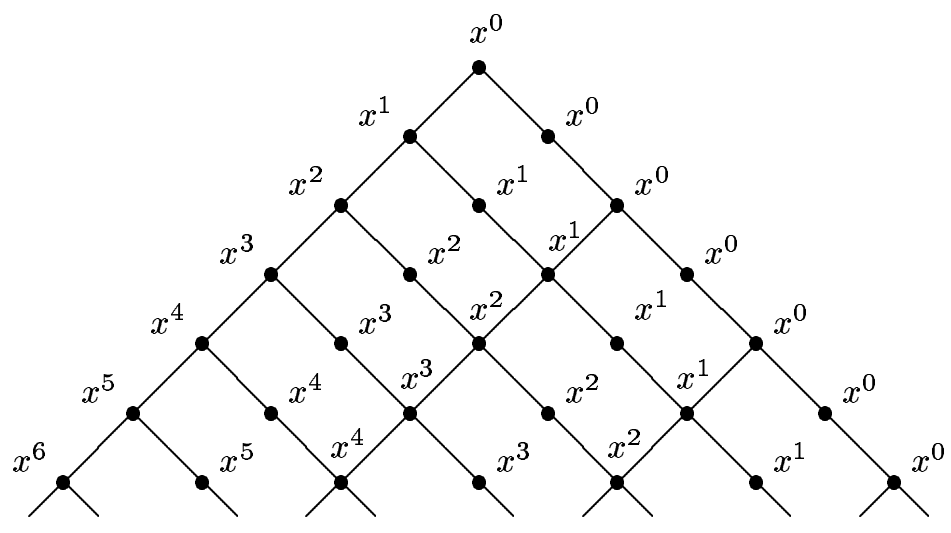

Рис. 1 
Коэффициенты разложения $G_{k}(x)$ по степеням $x$ могут быть легко вычислены по этому графу. На $k$-м уровне (т.е. на расстоянии $k$ от корневой вершины, $k=0,1,2, \ldots$ ) в графе имеется $k+1$ вершин (все ребра графа имеют длину 1). Каждой из этих вершин приписана некоторая степень $x$ : первой слева степень $x^{k}$, второй слева $-x^{k-1}$ и т.д., самой правой $-x^{0}$. Значение модуля коэффициента при $x^{j}$ равно числу путей длины $k$ из корневой вершины в ту вершину $k$-го уровня, которой приписана степень $x^{j}$. Знак коэффициента также определяется просто: на $k$-м уровне при $x^{k}$ стоит знак '+', при двух следующих степенях знак '-', затем снова дважды знак '+' и т.д. Проделав указанные выкладки и просуммировав их результаты по всем вершинам $k$-го уровня, получим формулу (20) с заменой $1+\lambda$ на $x$.

Теперь уже мы можем сформулировать основной результат этого пункта.

Теорема 1. Пусть $Q=q^{m}$, где $m=2 k+1, k \geqslant 2, q=p^{\ell}$ и $p$-нечетное простое число. Тогда модуль тригонометрической суммы многочлена

$$
f(x)=a_{0} x^{2}+2 \sum_{s=1}^{k-1} a_{s} x^{1+q^{s}}
$$

с коэффичиентами из $F_{q}$ достигает оченки Вейля

$$
|S(f)|=q^{m-3 / 2}
$$

если и только если все әлементы $a_{s}$ определяются равенствами

$$
a_{s}=\frac{\sum_{j=s}^{k-1}(-1)^{k-1-j} G_{j}\left(1+\lambda_{0}\right)}{G_{k-1}\left(1+\lambda_{0}\right)} a_{k-1}, \quad s=0,1, \ldots, k-2,
$$

где $a_{k-1}$ - произвольный ненулевой әлемент поля $F_{q}, a \lambda_{0} \in F_{q}-$ произвольный корень уравнения

$$
G_{k}(1+\lambda)=0 \text {. }
$$

Число различных таких многочленов $f(x)$ равно $(q-1) \mu_{k}$, где $\mu_{k}-$ число различных решений уравнения $(22)$ в поле $F_{q}$.

ДокАЗАТЕльство. Фактически надо лиш непосредственно проверить, что определяемые равенствами (21) коэффициенты $a_{s}$ удовлетворяют рекуррентньм соотношениям (10) при $\lambda=\lambda_{0}$, ибо соотношение (11) согласно утверждению 2 эквивалентно равенству $G_{k}\left(1+\lambda_{0}\right)=0$. Заметим лишш, что правые части равенств (21) определены, так как два соседних многочлена $G_{i+1}(x)$ и $G_{i}(x)$ не могут одновременно обращаться в нуль в одной и той же точке $x$ и, следовательно, $G_{k-1}\left(1+\lambda_{0}\right) \neq 0$.

Приведем в качестве следствия простой пример.

СлЕДСТВИЕ 1. Пусть $Q=q^{m}$, где $m=2 k+1, q=p^{\ell}$ и характеристика поля $p$ делит $2 k+1$. Тогда модуль тригонометрической суммы многочлена

$$
f(x)=a x^{2}+2 a \sum_{s=1}^{k-1}\left(1-4 s^{2}\right) x^{1+q^{s}},
$$

где а - произвольный ненулевой әлемент поля $F_{q}$, достигает оценки Вейля

$$
|S(f)|=q^{m-3 / 2} \text {. }
$$


ДоКАЗАТЕЛЬСТВо. Положим $\lambda_{0}=-3$. При этом

$$
G_{k}\left(1+\lambda_{0}\right)=G_{k}(-2)=(-1)^{k}(2 k+1)=0
$$

ибо $p$ делит $2 k+1$ (величина $G_{k}(-2)$ легко вычисляется по рекуррентному соотношению (12)). Коэффициенты многочлена вычисляются по формуле (21):

$$
a_{s}=\frac{k^{2}-s^{2}}{2 k-1} \cdot a_{k-1}=\left(1-4 s^{2}\right) a, \quad s=0,1, \ldots, k-2,
$$

где $a=a_{k-1} /(4(2 k-1))$.

3. Случай $m=2 k, k \geqslant 3$. Для четного $m=2 k$ многочлен вида (5) максимальной степени $q^{k}+1$ не может достигать оценки Вейля (2), вырождающейся в этом случае в тривиальную (равную $q^{m}$ ), ибо согласно (4) наибольшее значение модуля тригонометрической суммы такого многочлена равно $q^{m-1 / 2}$ и достигается оно на тех многочленах, для которых ранг матрицы $B$ равен 1 . Все такие многочлены были описаны в работе [2, теорема 3]. Кроме того, в этой же работе были изучены два крайних случая достижения оценки Вейля: $t=k-1$ (т.е. ранг матрицы $B$ равен 2 ) и $t=1$ (т.е. ранг матрицы $B$ равен $2 k-2)$, и описаны все многочлены вида (5) степени $q^{k-1}+1$ и $q+1$, при условии, что коэффициенты многочлена принадлежат полю $F_{q}$, а не полю $F_{q^{m}}$ (см. [2, теоремы 4 и 5]). Теперь мы рассмотрим случай, когда $t=k-2$ (т.е. матрица $B$ имеет ранг 4$)$, при том же условии на коэффициенты: $a_{0}, a_{1}, \ldots, a_{k-2}$ принадлежат полю $F_{q}$. Тогда первые пять строк матрицы $B$ имеют вид $\left(a_{k}=a_{k-1}=0, a_{k-2} \neq 0\right)$

$$
\left[\begin{array}{cccccccccccccc}
a_{0} & a_{1} & a_{2} & \ldots & a_{k-2} & 0 & 0 & 0 & a_{k-2} & a_{k-3} & a_{k-4} & \ldots & a_{2} & a_{1} \\
a_{1} & a_{0} & a_{1} & \ldots & a_{k-3} & a_{k-2} & 0 & 0 & 0 & a_{k-2} & a_{k-3} & \ldots & a_{3} & a_{2} \\
a_{2} & a_{1} & a_{0} & \ldots & a_{k-4} & a_{k-3} & a_{k-2} & 0 & 0 & 0 & a_{k-2} & \ldots & a_{4} & a_{3} \\
a_{3} & a_{2} & a_{1} & \ldots & a_{k-5} & a_{k-4} & a_{k-3} & a_{k-2} & 0 & 0 & 0 & \ldots & a_{5} & a_{4} \\
a_{4} & a_{3} & a_{2} & \ldots & a_{k-6} & a_{k-5} & a_{k-4} & a_{k-3} & a_{k-2} & 0 & 0 & \ldots & a_{6} & a_{5}
\end{array}\right]
$$

Если пятая строка $B$ является линейной комбинацией первых четырех строк, то ее ранг равен 4 , так как матрица $B$ циклическая, а ее первые четыре строки линейно независимы. Обозначим через $b_{i}$ строку $B$ с номером $i$. Пятая строка $b_{5}$ матрицы $B$ является линейной комбинацией первых четырех тогда и только тогда, когда найдутся элементы $\lambda_{1}, \lambda_{2}, \lambda_{3}, \lambda_{4} \in F_{q},\left(\lambda_{1}, \lambda_{2}, \lambda_{3}, \lambda_{4}\right) \neq(0,0,0,0)$ такие, что

$$
b_{5}=\lambda_{1} b_{1}+\lambda_{2} b_{2}+\lambda_{3} b_{3}+\lambda_{4} b_{4}
$$

Обозначим через $B_{5}$ матрицу, образованную первыми пятью строками матрищы $B$. Рассматривая столбцы матрицы $B_{5}$ с тремя нулями (т.е. столбцы с номерами $k+2, k+3$ и $k+4)$ и столбцы с двумя нулями (т.е. столбцы с номерами $k+1$ и $k+5)$, из соотношения (23) находим, что

$$
\begin{aligned}
a_{k-3} & =\lambda_{4} a_{k-2}, \\
a_{k-2} & =\lambda_{1} a_{k-2}, \\
0 & =\lambda_{1} a_{k-3}+\lambda_{2} a_{k-2}, \\
a_{k-4} & =\lambda_{4} a_{k-3}+\lambda_{3} a_{k-2}, \\
0 & =\lambda_{1} a_{k-4}+\lambda_{2} a_{k-3}+\lambda_{3} a_{k-2} .
\end{aligned}
$$


Отсюда получаем (так как $a_{k-2} \neq 0$ )

$$
\lambda_{1}=1, \quad \lambda_{4}=-\lambda_{2}, \quad \lambda_{3}=0
$$

Полагая $\lambda_{2}=\lambda$, перепишем соотношение (23) в следующем виде:

$$
b_{1}=-\lambda b_{2}+\lambda b_{4}+b_{5}
$$

С учетом (25) последние $k-3$ столбцов матрицы $B_{5}$ позволяют выразить все коэффициенты $a_{i}, i=1, \ldots, k-3$, через $a_{k-2}$ и $\lambda$ с помощью следующих рекуррентных соотношений:

$$
\begin{aligned}
& a_{k-3}=-\lambda a_{k-2}, \\
& a_{k-4}=-\lambda a_{k-3} \text {, } \\
& a_{k-5}=-\lambda a_{k-4}+\lambda a_{k-2}, \\
& a_{k-6}=-\lambda a_{k-5}+\lambda a_{k-3}+a_{k-2}, \\
& a_{i}=-\lambda a_{i+1}+\lambda a_{i+3}+a_{i+4}, \quad i=1, \ldots, k-6 .
\end{aligned}
$$

Рассмотрим теперь первые пять столбцов матрищы $B_{5}$ (остальные столбцы дают те же соотношения, что и (26)). Нетрудно видеть, что первьй столбец порождает то же соотношение, что и пятьй, а второй - то же, что и четвертьй (соотношение, задаваемое третьим столбцом, автоматически вьполняется). Из первого столбца $B_{5}$ вытекает, что рекуррентное соотношение (26) имеет место и при $i=0$.

Итак, все коэффициенты $a_{i}, i=0,1, \ldots, k-3$, выражаются через $a_{k-2}$ и $\lambda$, и осталось еще одно соотношение, порождаемое вторым столбцом матрицы $B_{5}$ :

$$
a_{3}=\lambda a_{0}+a_{1}-\lambda a_{2}
$$

Это соотношение легко переписывается в простом эквивалентном виде.

УТВЕРЖДЕНИЕ 3. Соотношение (27) әквивалентно соотношению

$$
E_{k-1}(\lambda)=0
$$

где $E_{k-1}(\lambda)$ - многочлен Диксона второго рода степени $k-1$.

ДоКАЗАТЕЛЬСТВО полностью аналогично доказательству утверждения 2 , и поэтому мы его опускаем. Напомним только, что многочлен $E_{k}(x)$ удовлетворяет рекуррентному соотношению (12) с начальными условиями (19) и определяется равенством (18).

ЗАмЕчАниЕ 2. Естественно, что формула (18), как и формула (20), имеет графовую интерпретацию. Как и в замечании 1 , рассматривается плоский граф-решетка, ребра которого по одной оси имеют длину 1 , а по другой - длину 2 , но не добавляется никаких 


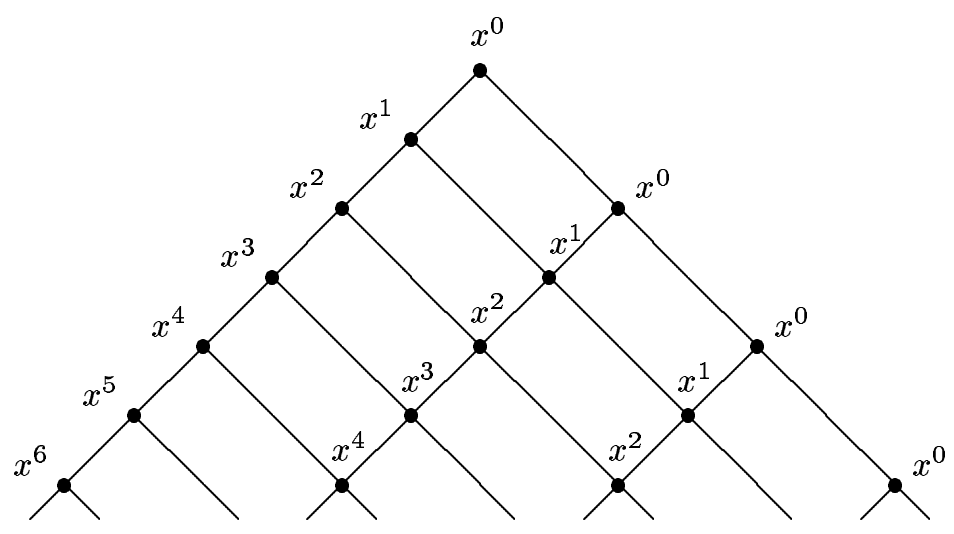

Рис. 2

новых вершин (так что степень корневой вершины равна 2, степень вершин, расположенных на осях, равна 3 и остальных вершин равна 4). Этот граф изображен на рис. 2.

Коэффициенты разложения $E_{k}(x)$ по степеням $x$ могут быть легко вычислены по этому графу. На $k$-м уровне (т.е. на расстоянии $k$ от корневой вершины, $k=0,1,2, \ldots$ ) в графе имеется $\lceil k / 2\rceil$ вершин (ребра, параллельные левой оси, имеют длину 1 , а параллельные правой - длину 2), каждой из которых приписана некоторая степень $x$ : первой слева степень $x^{k}$, второй $-x^{k-2}$, третьей $-x^{k-4}$ и т.д. Значение модуля коэффициента при $x^{j}$ равно числу путей длины $k$ из корневой вершины в ту вершину $k$-го уровня, которой приписана степень $x^{j}$. Знак коэффициента также определяется просто: на $k$-м уровне при $x^{k}$ стоит знак '+', при $x^{k-2}$ степени знак '-', затем при $x^{k-4}$ снова знак ' + ' и т.д. Проделав указанные выкладки и просуммировав их результаты по всем вершинам $k$-го уровня, получим формулу (18).

Теперь уже мы можем сформулировать основной результат этого пункта.

Теорема 2. Пусть $Q=q^{m}$, где $m=2 k, k \geqslant 3, q=p^{\ell}$ u $p$ - нечетное простое число. Тогда модуль тригонометрической суммы многочлена с коэффициентами из $F_{q}$

$$
f(x)=a_{0} x^{2}+2 \sum_{s=1}^{k-2} a_{s} x^{1+q^{s}}
$$

достигает оченки Вейля

$$
|S(f)|=q^{m-2}
$$

если и только если все әлементы $a_{s}$ определяются равенствами

$$
\begin{aligned}
a_{k-2 t} & =\sum_{j=0}^{t-1} E_{2 j}\left(\lambda_{0}\right) a_{k-2}, & t & =1,2, \ldots,\left\lfloor\frac{k}{2}\right\rfloor, \\
a_{k-2 t-1} & =-\sum_{j=0}^{t-1} E_{2 j+1}\left(\lambda_{0}\right) a_{k-2}, & & t=1,2, \ldots,\left\lfloor\frac{k-1}{2}\right\rfloor,
\end{aligned}
$$


где $a_{k-2}$ - произвольный ненулевой әлемент поля $F_{q}$, a $\lambda_{0} \in F_{q}-$ произвольный корень уравнения (28). Число различных таких многочленов $f(x)$ равно $(q-1) \mu_{k}$, где $\mu_{k}$ - число различных решений уравнения (28) в поле $F_{q}$.

ДокАЗАТЕЛЬСТво. Фактически надо лишш непосредственно проверить, что определяемые равенствами (29) и (30) коэффициенты $a_{s}$ удовлетворяют рекуррентньг соотношениям (26) при $\lambda=\lambda_{0}$, ибо соотношение (27) согласно утверждению 3 эквивалентно равенству $E_{k}\left(\lambda_{0}\right)=0$.

Приведем в качестве следствия простой пример.

СледСТВИЕ 2. Пусть $Q=q^{m}$, где $m=2 k, k \geqslant 3, q=p^{\ell}$ u характеристика поля $p$ делит $k+1$. Тогда модуль тригонометрической суммы многочлена

$$
f(x)=\frac{1}{8} a\left(1+(-1)^{k}\right) x^{2}+2 a \sum_{t=1}^{\lfloor k / 2\rfloor} t^{2} x^{1+q^{k-2 t}}-2 a \sum_{t=1}^{\lfloor(k-1) / 2\rfloor} t(t+1) x^{1+q^{k-2 t-1}}
$$

где $а$ - произвольный ненулевой әлемент поля $F_{q}$, достигает оценки Вейля

$$
|S(f)|=q^{m-2}
$$

ДоКАЗАТЕЛЬСТВо. Положим $\lambda_{0}=2$. При этом $E_{k}(2)=k+1=0$, ибо $p$ делит $k+1$ (величина $E_{k}(2)$ легко вычисляется по рекуррентному соотношению (12)). Коэффищиенты многочлена вычисляются по формулам (29) и (30):

$$
\begin{aligned}
a_{k-2 t} & =\sum_{j=0}^{t-1} E_{2 j}(2) a_{k-2}=a t^{2}, & t=1,2, \ldots,\left\lfloor\frac{k}{2}\right\rfloor, \\
a_{k-2 t-1} & =-\sum_{j=0}^{t-1} E_{2 j+1}(2) a_{k-2}=-a t(t+1), & t=1,2, \ldots,\left\lfloor\frac{k-1}{2}\right\rfloor,
\end{aligned}
$$

где $a=a_{k-2}$.

\section{СПИСОК ЦИТИРОВАННОЙ ЛИТЕРАТУРЫ}

[1] Лидл Р., Нидеррайтер Г. Конечные поля. М.: Мир, 1988.

[2] Бассалыго Л.А., Зиновьев В. А. Многочлены специального вида над конечным полем с максимальным модулем тригонометрической суммы // УМН. 1997. Т. 52. № 2. С. 31-44.

[3] Малышев А. В. О представлении целых чисел положительньми квадратичными формами. Тр. МИАН. Т. 65. М.: Наука, 1962.

[4] Бассалыго Л.А., Зиновьев В. А. О многочленах над конечным полем четной характеристики с максимальным значением модуля тригонометрической суммы // Матем. заметки. 2002. T. 72. № 2. C. 171-177.

[5] Lidl R., Mullen G. L., Turnwald G. Dickson Polynomials. Pitman Monographs and Surveys in Pure and Applied Mathematics. V. 65. New York: John Wiley and Sons, 1993. 